\title{
Fault Detection of Networked Systems Based on Passivity
}

\author{
Dongliang $\mathrm{Wu}^{1, \mathrm{a}}$, Yao Feng ${ }^{1, \mathrm{~b}}$ and Tao Liao ${ }^{1, \mathrm{c}}$ \\ ${ }^{1}$ Beijing Institute of Spacecraft Environment Engineering, Beijing, 100094, China \\ awudongliang.xidian@163.com, ${ }^{\text {b }}$ fenggyao@hotmail.com, ${ }^{\mathrm{c}}$ liaotao268@hotmail.com
}

Keywords: Fault detection; networked systems; passivity.

\begin{abstract}
This paper investigates the fault detection problem for networked systems based on passivity theory. By developing the filtering-based residual generator satisfying passivity, sufficient conditions can be established for ensuring that the fault can be detected in time. Finally, an illustrative example is provided to demonstrate the effective of the theoretical results.
\end{abstract}

\section{Introduction}

Networked control systems (NCSs) have gained tremendous attentions during the last decade and have wide applications in various fields [1,2]. By applying the communication network, the performance of control systems can be considerably improved and lower cost can be obtained accordingly. As a consequence, there have been a lot of effective analysis and synthesis approaches for NCSs. It should be pointed out that certain fault may happen in the NCSs, such that fault detection problem for NCSs is very important in both theory and real-world applications $[3,4,5]$. Note that filtering-based fault detection methods are useful and achieved many remarkable results.

On the other hand, passivity theory has been applied in kinds of complex systems due to its powerful framework. With passivity performance, system inputs and outputs can be related with respect to energy issues. For control systems, there are many efforts on passivity theory, which can show great advantages [6,7]. However, it is worth mentioning that there are few results on the fault detection problem for NCSs based on passivity theory, which motivates us for this paper.

In this paper, the passivity performance is introduced for the fault detection problem of NCSs. We first build the filtering-based residual generator, then we develop the sufficient conditions based on Lyapunov-Krasovskii functional for the augmented error system ensuring the passivity, which means that the fault can be detected.

The remainder is arranged as follows: the preliminaries are given for NCSs and the corresponding fault detection problem is formulated. Then, the main results of our paper are presented in the form of linear matrix inequalities. Moreover, we give a numerical example for demonstrating our theoretical results. In the end, the paper is concluded with future perspectives.

We use the following notations: * denotes the ellipsis for symmetry terms in matrices and $\operatorname{diag}\{*\}$ denotes the block-diagonal matrices.

\section{Problem Formulation and Preliminaries}

We are considering the following class of NCSs:

$$
\left\{\begin{array}{l}
\dot{x}(t)=A x(t)+B u(t)+B_{\omega} \omega(t)+B_{f} f(t), \\
y(t)=C x(t),
\end{array}\right.
$$

where $x(t)$ denotes the system state, $u(t)$ denotes the control input, $\omega(t)$ denotes the external disturbance and $f(t)$ denotes the system fault to be detected, $y(t)$ is the measurement output. Matrices $A, B, B_{\omega}, B_{f}$ and $C$ are known and constant.

In order to generate the residual signal, we develop a filter-type residual generator as follows: 


$$
\left\{\begin{array}{l}
\dot{x}_{F}(t)=A_{F} x_{F}(t)+B_{F} y(t), \\
r_{F}(t)=C_{F} x_{F}(t)+D_{F} y(t),
\end{array}\right.
$$

where $x_{F}(t)$ denotes the generator state and $r_{F}(t)$ denotes the generator output.

Considering the network environment, $y(t)$ is sampled and held with $\mathrm{ZOH}$ as $y\left(t_{k}\right)$, where $t_{k}$ denotes the sampling times by sampling period of $h$. Then, one has

$$
\left\{\begin{array}{l}
\dot{x}_{F}(t)=A_{F} x_{F}(t)+B_{F} y\left(t_{k}\right), \\
r_{F}(t)=C_{F} x_{F}(t)+D_{F} y\left(t_{k}\right) .
\end{array}\right.
$$

In addition, the weighting fault signal $W_{f}(t)$ is adopted which satisfies that

$$
\left\{\begin{array}{l}
\dot{x}_{f}(t)=A_{w} x_{f}(t)+B_{W} f(t), \\
r_{f}(t)=C_{W} x_{f}(t)+D_{W} f(t),
\end{array}\right.
$$

where $r_{f}(t)$ is the reference residual signal.

Denote $r(t)=r_{F}(t)-r_{f}(t)$ and $e(t)=x(t)-x_{F}(t)$. Then, it can be obtained that

$$
\left\{\begin{array}{l}
\dot{\zeta}(t)=\tilde{A} \zeta(t)+\tilde{A}_{k} \zeta\left(t_{k}\right)+\tilde{B} \varpi(t), \\
r(t)=\tilde{C} \zeta(t)+\tilde{C}_{k} \zeta\left(t_{k}\right)+\tilde{D} \varpi(t),
\end{array}\right.
$$

where $\zeta(t)=\left[x^{T}(t), e^{T}(t), x_{f}^{T}(t)\right]^{T}$ and $\varpi(t)=\left[u^{T}(t), \omega^{T}(t), f^{T}(t)\right]^{T}$ with

$$
\begin{aligned}
& \tilde{A}=\left[\begin{array}{ccc}
A & 0 & 0 \\
A-A_{F} & A_{F} & 0 \\
0 & 0 & A_{w}
\end{array}\right], \tilde{A}_{k}=\left[\begin{array}{ccc}
0 & 0 & 0 \\
-B_{F} C & 0 & 0 \\
0 & 0 & 0
\end{array}\right], \\
& \tilde{B}=\left[\begin{array}{ccc}
B & B_{\omega} & B_{f} \\
B & B_{\omega} & B_{f} \\
0 & 0 & B_{W}
\end{array}\right], \tilde{C}=\left[\begin{array}{lll}
C_{F} & -C_{F} & -C_{W}
\end{array}\right], \\
& \tilde{C}_{k}=\left[\begin{array}{lll}
D_{F} C & 0 & 0
\end{array}\right], \tilde{D}=\left[\begin{array}{lll}
0 & 0 & -D_{W}
\end{array}\right] .
\end{aligned}
$$

Denote the threshold $J_{t h}$ and residual evaluation function $J_{t h}$ as follows:

$$
\left\{\begin{array}{l}
J_{t h}=\int_{t_{0}}^{t_{0}+t^{*}} r_{r}^{T}(t) r_{r}(t) d t, \\
J(r)=\int_{t_{0}}^{t_{0}+t^{*}} r_{r}^{T}(t) r_{r}(t) d t,
\end{array}\right.
$$

where $t_{0}$ represents the initial time and $t^{*}$ represents the evaluation time.

Consequently, one can obtain that

$$
\left\{\begin{array}{l}
J(r)>J_{t h}, \text { NCSs with faults, } \\
J(r) \leq J_{t h}, \text { NCSs with no faults. }
\end{array}\right.
$$

To this end, the following defintion is given with passivity performance: the system in Eq. 5 can satisfy the passivity performance if $2 \int_{0}^{t_{p}}\left(r^{T}(t) \varpi(t) d t \geq-\gamma \int_{0}^{t_{p}} \varpi^{T}(t) \varpi(t) d t\right.$. 


\section{Main Results}

In this section, we will design the filter gains of the residual generator with the help of linear matrix inequalities.

Theorem 1. The fault of NCS in Eq. 1 can be detected with the given filter gains $A_{F}, B_{F}, C_{F}$ and $D_{F}$, if there exist symmetric matrices $P>0, R>0$ such that the following linear matrix inequalities can hold:

$$
\left[\begin{array}{ccccc}
P \tilde{A}+\tilde{A}^{T} P-R+\tilde{C}^{T} \tilde{C}+ & P \tilde{A}_{k}+R & 0 & P \tilde{B}-\tilde{C} & h \tilde{A}^{T} R \\
* & -2 R & R & -\tilde{C}_{k} & h \tilde{A}_{k}^{T} R \\
* & * & -R & 0 & 0 \\
* & * & * & -\gamma I-2 \tilde{D} & h \tilde{B}^{T} R \\
* & * & * & * & -R
\end{array}\right]<0
$$

Proof. Choose the Lyapunov-Krasovskii candidate by

$$
\mathrm{V}(\mathrm{t})=\zeta^{T}(t) P \zeta(t)+h \int_{-h}^{0} \int_{t+\varphi}^{t} \dot{\zeta}^{T}(\eta) R \dot{\zeta}(\eta) d \eta d \varphi .
$$

Then, the proof can be derived directly with the input delay approach when $V(\mathrm{t})>0$ and $\dot{V}(\mathrm{t})<0$.

Theorem 2. The fault of NCS in Eq. 1 can be detected with the filter gains $A_{F}, B_{F}, C_{F}$ and $D_{F}$, if there exist $P=\operatorname{diag}\left\{P_{1}, P_{2}, P_{3}\right\}>0, R=\operatorname{diag}\left\{R_{1}, R_{2}, R_{3}\right\}>0$ and matrices $\bar{A}_{F}, \bar{B}_{F}, \bar{C}_{F}, \bar{D}_{F}$, such that the following linear matrix inequalities can hold:

$$
\Pi=\left[\begin{array}{cc}
\Pi_{1} & \Pi_{2} \\
* & \Pi_{3}
\end{array}\right]<0
$$

where

$$
\begin{aligned}
& \Pi_{1}=\left[\begin{array}{ccc}
2 P_{1} A-R_{1} & A^{T} P_{2}-\bar{A}_{F}^{T} & 0 \\
* & 2 \bar{A}_{F}-R_{2} & 0 \\
* & * & 2 P_{3} A_{w}-R_{3}
\end{array}\right]\left[\begin{array}{rrr}
R_{1} & 0 & 0 \\
-\bar{B}_{F} C & R_{2} & 0 \\
0 & 0 & R_{3}
\end{array}\right] \quad \begin{array}{r}
0 \\
-2 R
\end{array} \\
& \Pi_{2}=\left[\begin{array}{cc}
{\left[\begin{array}{ccc}
P_{1} B-C_{F}^{T} & P_{1} B_{\omega}-C_{F}^{T} & P_{1} B_{f}-C_{F}^{T} \\
P_{2} B-C_{F}^{T} & P_{2} B_{\omega}-C_{F}^{T} & P_{2} B_{f}-C_{F}^{T} \\
-C_{W}^{T} & -C_{W}^{T} & P_{3} B_{W}-C_{W}^{T}
\end{array}\right]} & h\left[\begin{array}{ccc}
A^{T} P_{1} & A^{T} P_{2}-\bar{A}_{F}^{T} & 0 \\
0 & \bar{A}_{F}^{T} & 0 \\
0 & 0 & A_{W}^{T} P_{3}
\end{array}\right] \\
-\left[\begin{array}{ccc}
C^{T} D_{F}^{T} & C^{T} D_{F}^{T} & C^{T} D_{F}^{T} \\
0 & 0 & 0 \\
0 & 0 & 0
\end{array}\right] & h\left[\begin{array}{ccc}
0 & -C^{T} \bar{B}_{F}^{T} & 0 \\
0 & 0 & 0 \\
0 & 0 & 0
\end{array}\right]
\end{array}\right],
\end{aligned}
$$

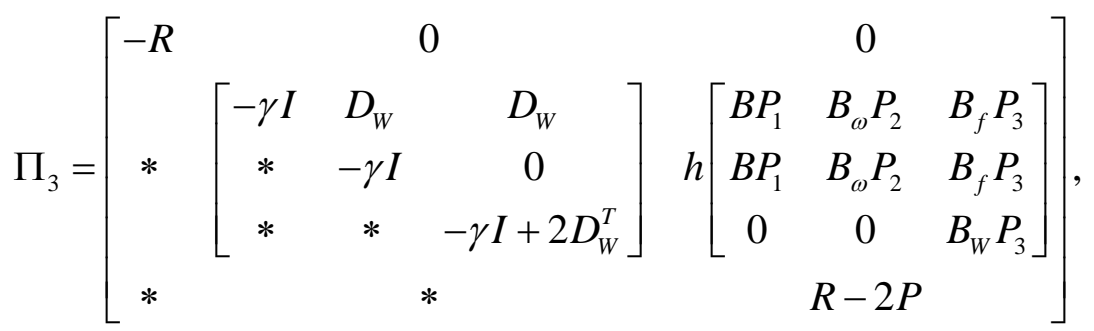


and the filter gains can be designed by

$$
\left[\begin{array}{cc}
A_{F} & B_{F} \\
C_{F} & D_{F}
\end{array}\right]=\left[\begin{array}{cc}
P_{2}^{-1} & 0 \\
0 & I
\end{array}\right]\left[\begin{array}{ll}
\bar{A}_{F} & \bar{B}_{F} \\
\bar{C}_{F} & \bar{D}_{F}
\end{array}\right]
$$

Proof. Let $P_{2} A_{F}, P_{2} B_{F}, C_{F}$ and $D_{F}$ denote as $\bar{A}_{F}, \bar{B}_{F}, \bar{C}_{F}$ and $\bar{D}_{F}$, the proof can follow directly from Theorem 1 .

\section{Illustrative Example}

In this section, we give a numerical example to show the effectiveness of our proposed method.

Given the parameters in Eq. 1 as follows:

$$
A=\left[\begin{array}{cc}
-1.5 & 1 \\
0 & -4
\end{array}\right], B=\left[\begin{array}{c}
0.4 \\
-0.4
\end{array}\right], B_{\omega}=\left[\begin{array}{l}
0.3 \\
0.3
\end{array}\right], B_{f}=\left[\begin{array}{l}
0.5 \\
0.3
\end{array}\right], C=\left[\begin{array}{cc}
1 & 0.5 \\
0.2 & 0.4
\end{array}\right] .
$$

Set $u(t)=3+\cos t, f(t)=2$ as $3 \leq t \leq 5$ and $\omega(t)$ as band-limited white noise. As a result, the desired filtering gains can be designed as follows:

$$
\begin{aligned}
A_{F} & =\left[\begin{array}{cc}
-0.6185 & 0.5208 \\
-0.1112 & -1.6627
\end{array}\right], B_{F}=\left[\begin{array}{cc}
-0.2446 & 0.3571 \\
0.0881 & -0.6894
\end{array}\right], \\
C_{F} & =\left[\begin{array}{ll}
1.7327 & 0.5836
\end{array}\right], D_{F}=\left[\begin{array}{ll}
-0.0547 & 0.1482
\end{array}\right] .
\end{aligned}
$$

With the designed residual generator, it can be verified that for $J(r)=\int_{0}^{3} r^{T}(t) r(t) d t=>J_{t h}$, which means that the fault can be detected after it occurs. Fig. 1 shows the residual evaluation function, which also supports the obtained results.

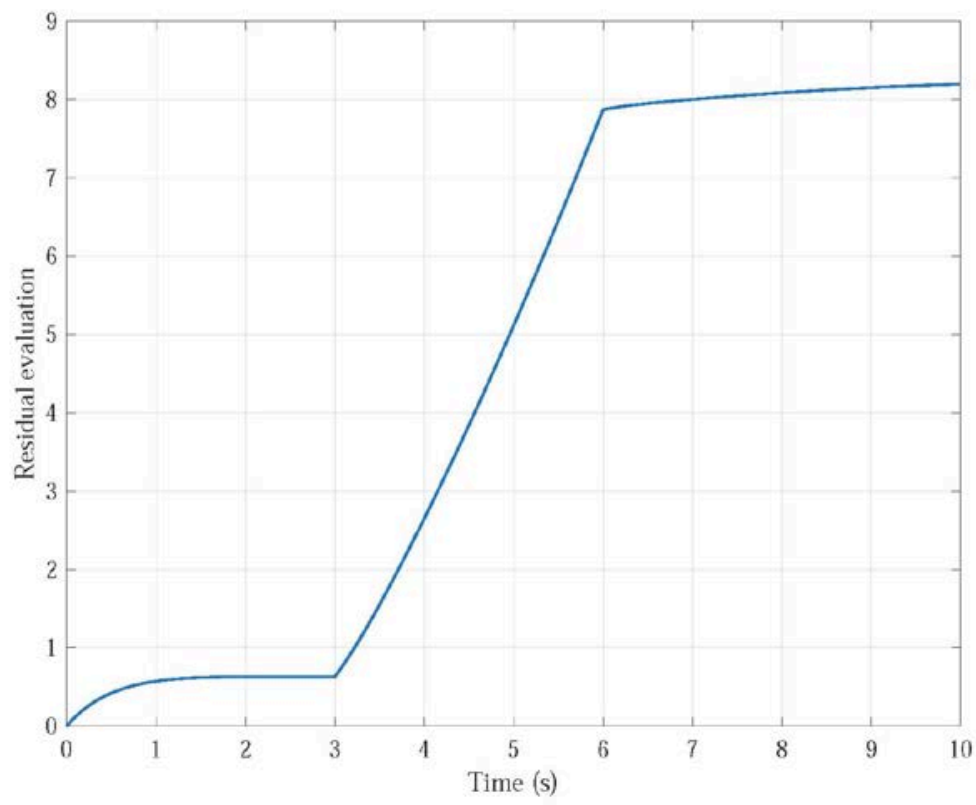

Fig. 1 The residual evaluation function

\section{Conclusions}

In this paper, we deal with the fault detection problem for NCSs by applying the passivity theory. The fault detection filter is developed for residual generation. Moreover, the design procedure for the corresponding filtering gains is given with sufficient conditions. In the end, the simulation results are 
given for demonstrating the correctness of our proposed fault detection method. One interesting future study may be extending the obtained results in this paper to the cases with bandlimited cases.

\section{References}

[1] R. A. Gupta and M. Y. Chow, IEEE transactions on industrial electronics, 2010, 57(7): 2527-2535.

[2] T. C. Yang, IEE Proceedings-Control Theory and Applications, 2006, 153(4): 403-412.

[3] R. Isermann, Automatica, 1984, 20(4): 387-404.

[4] H. Li, Z. Chen, L. Wu, et al, IEEE Transactions on Cybernetics, 2017, 47(4): 1041-1052.

[5] Y. L. Wang, T. B. Wang, and Q. L. Han, Information Sciences, 2016, 328: 577-594.

[6] Q. Hu, J. Li, and J. Zhang, Acta Astronautica, 2017, 131: 152-158.

[7] Z. G. Wu, P. Shi, Z. Shu, et al, IEEE Transactions on Automatic Control, 2017, 62(4): 2020-2025. 